Check for updates

Cite this: RSC Adv., 2017, 7, 50584

Received 12th September 2017 Accepted 18th October 2017

DOI: 10.1039/c7ra10130a

rsc.li/rsc-advances

\section{Preparation of nitrogen-doped carbon dots with high quantum yield from Bombyx mori silk for Fe(III) ions detection $\uparrow$}

\author{
Hui Liu, ${ }^{a}$ Yue Zhang, ${ }^{a}$ Jia Hui Liu, ${ }^{a}$ Peng Hou, ${ }^{a}$ Jun Zhou (D) *ab \\ and Cheng Zhi Huang ${ }^{D}$ *ac
}

\begin{abstract}
Nitrogen-doped carbon dots (CDs) have attracted increasing attention in the field of biochemistry and biosensors due to their high emission efficiency. Herein, Bombyx mori silk, natural fibres composed by two fibroin brins and conglutinated by sericin binder, has a high content of nitrogen, was used as raw material in the coupling of citric acid to prepare nitrogen-doped CDs using a facile one-step hydrothermal route. The as-prepared nitrogen-doped CDs are uniform with an average particle size of $5.6 \mathrm{~nm}$, emit blue fluorescence with the quantum yield of $61.1 \%$, and maintain photochemical stability in high salt concentrations and in most organic solvents. Binding experiments of the as-prepared CDs with metal ions show that the hydroxyl groups on their surface play very important role in the blue fluorescence emission, which can easily bind with $\mathrm{Fe}^{3+}$ as the consequence of fluorescence quenching, making a method for $\mathrm{Fe}^{3+}$ detection developed with high selectivity and sensitivity.
\end{abstract}

\section{Introduction}

Carbon dots (CDs) have attracted increasing attention since they were discovered as a new type of carbon nanomaterial. ${ }^{\mathbf{1 , 2}}$ Compared to conventional organic dyes and semiconductor quantum dots, ${ }^{3-5}$ CDs show high photostability, good biocompatibility, low cytotoxicity, easy preparation, and low cost. ${ }^{5-8}$ There is a trend that CDs become the optimal alternative to conventional fluorescent materials since CDs have begun to find its uses in fluorescent sensing and imaging, ${ }^{8-12}$ drug delivery and release, ${ }^{13-15}$ and other fields.

Up to now, new routes to prepared CDs with smart properties, ${ }^{\mathbf{1 6 - 2 0}}$ strategies for surface functionalization, ${ }^{21-26}$ and applications for bioimaging and biosensing ${ }^{7,27,28}$ have been well reported. It is noticeable that the initial discovered CDs have the quantum yield of generally less than $10 \%{ }^{29,30}$ With the exploration of new synthetic methods and improved routes, raw materials selection, and CDs surface modification ${ }^{31}$ or doping, ${ }^{32,33}$ the photoluminescent efficiency of CDs could be

${ }^{a}$ Key Laboratory of Luminescent and Real-Time Analytical Chemistry (Southwest University), Ministry of Education, College of Pharmaceutical Sciences, Southwest University, Chongqing 400716, China.E-mail: zhouj@swu.edu.cn; chengzhi@swu. edu.cn

${ }^{b}$ College of Computer and Information Science, Southwest University, Chongqing 400715, China

${ }^{c}$ Chongqing Key Laboratory of Biomedical Analysis (Southwest University), Chongqing Science \& Technology Commission, College of Chemistry and Chemical Engineering, Southwest University, Chongqing 400715, China

$\dagger$ Electronic supplementary information (ESI) available. See DOI: 10.1039/c7ra10130a significantly increased, making the applications of carbon dots expanded. Element doping, which can avoid complex modification processes, stands out from these strategies and can produce CDs with good emission fluorescence and special performance. For example, doping of nitrogen, since its sources are easy to access and the mechanism of action is related to the nitrogen-containing chromophore can passivate the active sites on the surface of the $\mathrm{CDs},{ }^{31}$ making the quantum yield substantially increased. Therefore, nitrogen doping is a very effective way to increase the quantum yield of CDs. ${ }^{34-37}$

Bombyx mori silk, a composite of natural proteins, has the nitrogen content as high as $18 \% .{ }^{38}$ Using Bombyx mori silk as a raw material, nitrogen-doped CDs have been prepared through a hydrothermal route and used as a fluorescent probe in bioimaging. ${ }^{39}$ However, the as-developed CDs merely have a photoluminescence quantum yield of $13.9 \%$, which are still low relative to those of semiconductor quantum dots. Furthermore, preparation of highly photoluminescent nitrogen-doped CDs by hydrothermal methods were reported in recent years. ${ }^{7,40}$ In addition, how to prepare CDs with high quantum yield and good biocompatibility, which has been scarcely studied in the literature. Therefore, the development of a facile, economic and green method to prepare CDs with high quantum yield and good biocompatibility using Bombyx mori silk remains challenging.

As Scheme 1 shown, we developed a facile and simple one step hydrothermal method to prepare blue fluorescent nitrogen-doped CDs with high quantum yield using Bombyx mori silk and citric acid. Citric acid was a carbon source to construct CDs, and silk was a nitrogen source and may serve as 


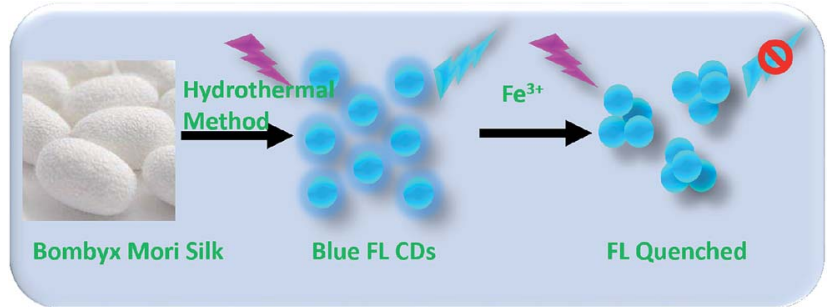

Scheme 1 Illustration of the formation process of highly luminescent nitrogen-doped CDs with blue $\mathrm{FL}$ and its further applications for $\mathrm{Fe}^{3+}$ detection. Using Bombyx mori silk and citric acid as raw materials, nitrogen-doped CDs with blue FL were prepared via hydrothermal method. Hydroxyl groups on the surface of the CDs can interact with $\mathrm{Fe}^{3+}$ which induces the aggregation of $\mathrm{CDs}$, resulting in $\mathrm{FL}$ quenching of CDs.

surface-passivating agent. In this work, the optical and chemical properties of as-prepared CDs were investigated by modern characterized techniques. The as-prepared CDs were homogeneous and had a certain crystal form that had an average particle size of $5.6 \mathrm{~nm}$, and a quantum yield as high as $61.1 \%$ was achieved. Meanwhile, the surface of the CDs was rich in carboxyl and hydroxyl groups, which enabled good water solubility and stable fluorescence properties. Furthermore, the hydroxyl groups on the surface of the as-prepared CDs are highly specific to $\mathrm{Fe}^{3+}$, which induces fluorescence quenching of CDs, making the as-prepared CDs as a luminescent probe for selective and sensitive detection of $\mathrm{Fe}^{3+}$.

\section{Experimental}

\section{Instruments and reagents}

A polytetrafluoroethylene reactor and an electric drying oven were purchased from Jinan Henghua Technology Co., Ltd., China and Shanghai Yiheng Scientific Instrument Co., Ltd., China, respectively. A fluorescence spectrophotometer (F-2500, Hitachi, Japan) was used for detecting the excitation and emission spectra of CDs. A UV-visible-near infrared spectrophotometer (UV-3600, Shimadzu, Japan) was used to detect the absorption spectrum of the aqueous solution of CDs. A highresolution transmission electron microscope (Tecnai G2 F20 S-TWIN, FEI, USA) was used to characterize the morphology, particle size, and lattice characteristics of the CDs with an accelerating voltage of $200 \mathrm{kV}$. A Dimension Icon ScanAsyst atomic force microscope was purchased from Brooks, USA. The hydrodynamic diameter and surface charge of the CDs were determined using a Zetasizer Nano instrument (Malvern Instruments, UK). Fourier transform infrared spectroscopy (FITR-8400S, Shimadzu), X-ray photoelectron spectroscopy (ESCALAB 250, Thermo, USA), and laser confocal Raman spectroscopy (LabRAM HR 800) were used to analyze the elements and functional groups of the CDs. A pH meter (PHS-3C, Chengdu Fangzhou Technology Development Co., China) was used to determine the acidity of the solutions. A vortex mixer (QL-9011, Jiangsu Linqibeier Instrument Manufacturing Co., Ltd.) was used to mix the solutions. A Canon $550 \mathrm{D}$ digital camera was used to take photos.
All of the solvents were analytical grade. Silk was provided by State Key Laboratory of Silkworm Genome Biology, Southwest University. $N, N$-Dimethylformamide (DMF) was purchased from Chengdu Kelong Reagent Co., Ltd. Polyacrylonitrile (PAN) was purchased from Sigma (Shanghai). Citric acid was purchased from Chuandong Chemical Reagent Co., Ltd. (Chongqing, China). The solutions used in the experiments were prepared using ultrapure water (18.2 $\mathrm{M} \Omega$ ).

\section{Preparation and purification of nitrogen-doped CDs}

$0.16 \mathrm{~g}$ of silk, $0.16 \mathrm{~g}$ of citric acid, and $4 \mathrm{~mL}$ of double distilled water were mixed in a $25 \mathrm{~mL}$ beaker, and then transferred to a $25 \mathrm{~mL}$ Teflon-lined stainless steel reactor. All of the silk was ensured to immerse in the solution, and then the steel set was installed and heated at $200{ }^{\circ} \mathrm{C}$ for $3.5 \mathrm{~h}$ in a drying oven. After the reaction, the reactor was cooled to room temperature. The product was centrifuged at $11000 \mathrm{rpm}$ for $15 \mathrm{~min}$ to wash away the incompletely reacted non-fluorescent substances, and the supernatant that contained the CDs was retained for further use. Subsequently, the remaining raw materials were removed via $12 \mathrm{~h}$ of dialysis with a dialysis bag that had a cutoff molecular weight of $500 \mathrm{D}$; the dialysate was replaced every $2 \mathrm{~h}$. Finally, a solid powder was obtained after freezing and vacuum drying.

\section{Calculation of the quantum yield}

Determination of relative fluorescence quantum yield was as follows. According to the literature ${ }^{29,41}$ quinine sulfate solution was used as the reference (quantum yield of 54\%), and the emission spectra of the CDs with quinine sulfate at different concentrations (excited at $360 \mathrm{~nm}$ ) and the corresponding absorption spectra were recorded. The absorbance values at $360 \mathrm{~nm}$ in the corresponding absorption spectra were then plotted in the $X$-direction, and the areas of the emission spectra were plotted in the $Y$-direction to calculate the slope of the curve. The quantum yield was then calculated according to the equation:

$$
\Phi_{\mathrm{x}}=\Phi_{\mathrm{ST}}\left(m_{\mathrm{x}} / m_{\mathrm{ST}}\right)\left(\eta_{\mathrm{x}}^{2} / \eta_{\mathrm{ST}}^{2}\right)
$$

where " $\Phi$ " represents the quantum yield, " $m$ " represents the slope of the areas of the emission spectra plotted against the absorbance values, and " $\eta$ " is the refractive index of the solvent (the refractive index of quinine sulfate is 1.33, and the refractive index of the CDs dissolved in double distilled water is 1.33). "ST" and " $\mathrm{x}$ " represent the standard and sample solutions, respectively.

\section{Detection of $\mathrm{Fe}^{3+}$ using nitrogen-doped CDs}

$0.25 \mathrm{mg}$ of fluorescent CDs powder prepared under the optimum conditions was dispersed in $1 \mathrm{~mL}$ of HAc-NaAc buffer solution with $\mathrm{pH} 3.2$ to prepare the fluorescent CDs solution with an actual concentration of $0.25 \mathrm{mg} \mathrm{mL}^{-1}$. Then $50 \mu \mathrm{L}$ of $0.25 \mathrm{mg} \mathrm{mL}{ }^{-1} \mathrm{CDs}$ and $50 \mu \mathrm{L}$ of $\mathrm{Fe}^{3+}$ solution at different concentrations were sequentially added to a $1.5 \mathrm{~mL} \mathrm{PE}$ tube, and the final volume was adjusted to $500 \mu \mathrm{L}$ by adding HAc- 
NaAc buffer with pH 3.2. The mixture was mixed with the vortex instrument and then left to stand for $10 \mathrm{~min}$ before the fluorescence determination. After the sample was evenly mixed, the fluorescence intensity was measured using an excitation wavelength of $360 \mathrm{~nm}$, and the experiment was conducted three times in parallel.

\section{Results and discussion}

\section{Preparation and characterization of CDs}

In this study, Bombyx mori silk and citric acid were used as the precursor to prepare nitrogen-doped CDs through one step hydrothermal route. Compared to the previously method to prepare CDs by using silk, ${ }^{39}$ we used citric acid as a carbon source to construct $\mathrm{CDs}^{42}$ and silk may serve as nitrogen sources ${ }^{39}$ and surface-passivating agent to achieve high quantum yield..$^{\mathbf{4 0 , 4 3 4 4}}$ The synthesized solid powder CDs dispersed in solution at an appropriate concentration was deposited on a copper grid and characterized using transmission electron microscopy (TEM) and atomic force microscopy (AFM), and the particle size and morphology of the CDs were analyzed using the results of these two methods. It can be seen from Fig. 1 that the resulting CDs were monodispersed spherical or nearly spherical shapes. The statistical chart in Fig. 1b, which was obtained from TEM image, showed that the sizes of the CDs were in the range of 4-6 $\mathrm{nm}$ with an average particle size of $5.6 \mathrm{~nm}$ and a narrow size distribution. In the HRTEM image (inset in Fig. 1a), it is observed that the CDs have a certain lattice structure, and the lattice space is $0.26 \mathrm{~nm}$, which is consistent with the parameters reported in the literature. The AFM imaging results of the CDs in Fig. 1c further reveals the morphology of the CDs. Fig. 1d shows the 3D height distribution of the CDs. The height was evenly distributed and
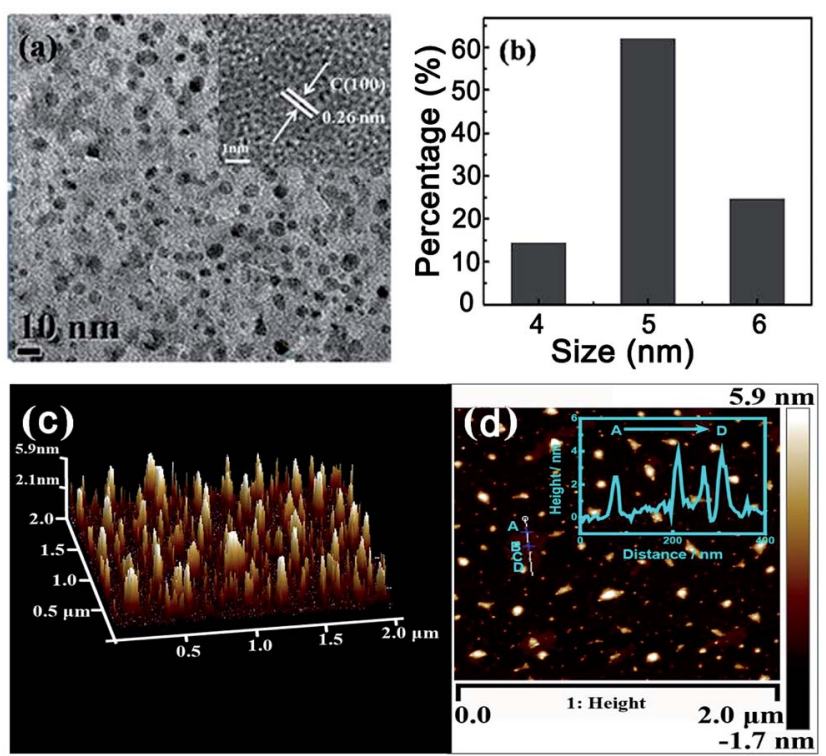

Fig. 1 Morphology characterization of the as-prepared CDs. (a) TEM image of CDs, (b) statistical distribution of the sizes of the CDs, (c) three-dimensional AFM image of the CDs, and (d) AFM image; inset: height distribution of the CDs. had a value of $4 \pm 0.5 \mathrm{~nm}$. In combination with the above TEM characterization, we can confirm that the CDs synthesized via this method were approximately spherical. In addition, the CDs were negatively charged $(-12.4 \mathrm{eV})$ according to zeta potential measurements.

\section{Structural characterization of CDs}

By analyzing the XPS spectrum of the CDs, the specific element composition can be obtained. Fig. 2a shows that the CDs mainly contained carbon, nitrogen, and oxygen. Among these elements, carbon had four different states, as shown in Fig. 2b. It can be seen from the $\mathrm{C}_{1 \mathrm{~s}}$ spectrum that the CDs had three distinct peaks at $284.6 \mathrm{eV}, 286.7 \mathrm{eV}$, and $288.1 \mathrm{eV}$, corresponding to $\mathrm{C}-\mathrm{C}, \mathrm{C}-\mathrm{O}-\mathrm{C}$, and $-\mathrm{COOH}$, respectively; the peak at $285.7 \mathrm{eV}$ corresponds to $\mathrm{C}-\mathrm{N}$. The nitrogen element can be classified into three categories: pyrrole-like nitrogen $(\mathrm{C}-\mathrm{N}-\mathrm{C})$ at $399.7 \mathrm{eV}$, graphite nitrogen or $\mathrm{N}-\mathrm{H}$ at $401.4 \mathrm{eV}$ (Fig. 2c), and $\mathrm{N}-$ $(\mathrm{C})_{3}$ at $402 \mathrm{eV}$. Three distinct peaks at $531.4 \mathrm{eV}, 532.1 \mathrm{eV}$, and $532.8 \mathrm{eV}$ can be observed in O1s spectrum of the CDs (Fig. 2d), which correspond to $\mathrm{C}-\mathrm{O}, \mathrm{C}=\mathrm{O}$, and $\mathrm{C}-\mathrm{OH} / \mathrm{C}-\mathrm{O}-\mathrm{C}$ groups. ${ }^{45}$

The above analysis results are consistent with the results of the FT-IR spectrum (Fig. 3a) and clearly demonstrate that the surface of the CDs was rich in functional groups such as hydroxyl, carboxyl, and amino groups. These hydrophilic functional groups enhance the water solubility and stability of the
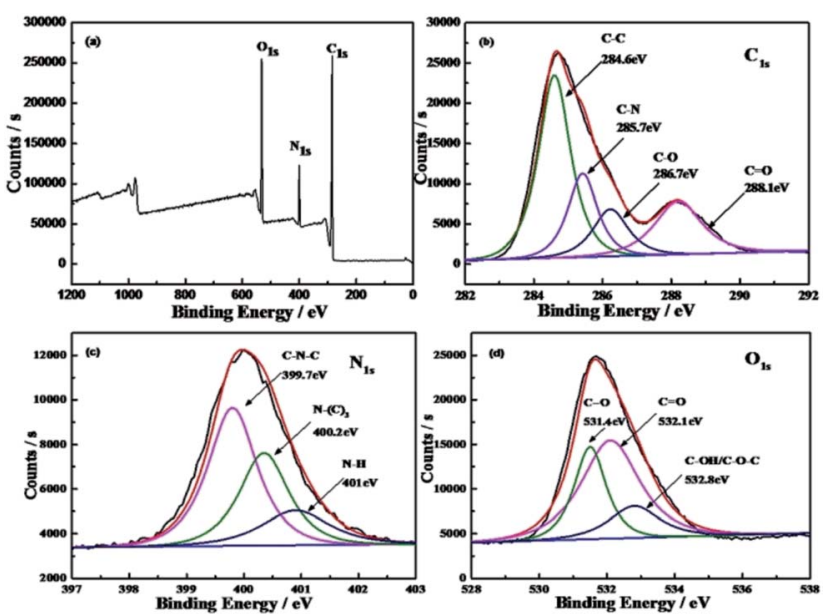

Fig. 2 XPS survey scan of the as-prepared CDs. (a) Survey, high resolution N1s (b), C1s (c), and O1s (d) XPS spectra of CDs.

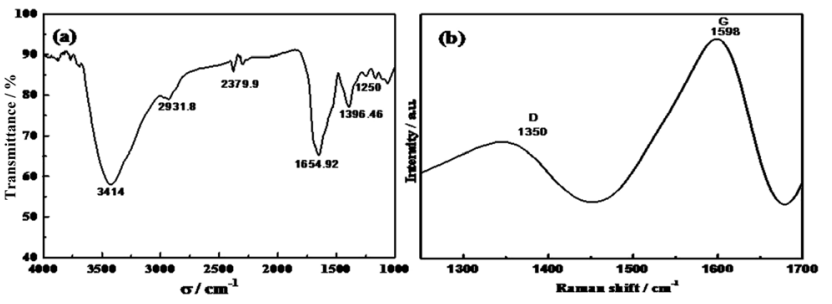

Fig. 3 FT-IR and Raman spectrum of the as-prepared CDs. (a) FT-IR spectrum of CDs, (b) Raman spectrum of CDs. 
CDs, which results in the CDs being highly dispersed and stabilized in aqueous solution. ${ }^{\mathbf{4 6}}$

To comprehensively explore the surface functional groups of the synthesized CDs and their structural components, we also carried out infrared and Raman spectroscopy and X-ray photoelectron spectroscopy characterization, and the results are shown in Fig. 3. In the FT-IR spectrum (Fig. 3a), there are two significant absorption peaks at $1654.92 \mathrm{~cm}^{-1}$ and $1396.46 \mathrm{~cm}^{-1}$, and these are attributed to the stretching vibration of $\mathrm{C}=\mathrm{O}$ and the stretching vibration of $\mathrm{C}-\mathrm{N}$, respectively. The absorption peak at $3414 \mathrm{~cm}^{-1}$ corresponds to the stretching vibration of $\mathrm{C}-\mathrm{O}$ in $-\mathrm{OH}$ and unsaturated hydroxyl groups, and this clearly illustrates the presence of both carboxyl and hydroxyl groups on the surface of the synthesized CDs. In addition, the absorption peak at $2931.8 \mathrm{~cm}^{-1}$ corresponds to the $\mathrm{C}-\mathrm{H}$ stretching vibration in alkanes.

To characterize the Raman spectroscopy, we deposited the CDs on a gold base and irradiated them with a $532 \mathrm{~nm}$ laser, and the spectrum is shown in Fig. $3 \mathrm{~b}$. There is a wide weak Dband peak at $1350 \mathrm{~cm}^{-1}$, indicating that there were $\mathrm{sp}^{3}$ hybridized carbons. The $\mathrm{G}$ band is located at $1589 \mathrm{~cm}^{-1}$ and indicates that there were $\mathrm{sp}^{2}$ hybridized carbons, which is associated with the plane vibration and is consistent with the results reported in the literature. ${ }^{47}$ In the X-ray diffraction (XRD) pattern of the CDs (Fig. S1 $\dagger$ ), there is a clear broad peak around $24^{\circ}$, and this is the result of the disordered carbon atoms. ${ }^{46}$ The internal structure and composition of the CDs were analyzed using the above characterization methods, and it can be concluded that the CDs synthesized via this method were mainly composed of $\mathrm{sp}^{2}$ hybridized carbons and that a portion of $\mathrm{sp}^{3}$ hybridized carbons was present in the form of defects.

In this assay, Bombyx mori silk and citric acid were used as the carbon source, and Bombyx mori silk was used as the nitrogen source. Citric acid can self-assemble and form carbon core nanosheet structures by carbonization. ${ }^{42}$ Carbon core state show relative low quantum yields. Heteroatom-doping and surface passivation can enhance the quantum yields of CDs. ${ }^{48}$ In $\mathrm{N}$-doped CDs, $\mathrm{N}$ atom enters carbon core and form pyrrolic structure (graphitic nitrogen), which effectively improves the quantum yields of N-doped CDs. ${ }^{49,50}$ Besides, the quantum yields of $\mathrm{N}$ doped CDs increase as the $\mathrm{N}$ content increase. ${ }^{48,51}$ From the XPS data, we can see that the $\mathrm{C}$ content and $\mathrm{N}$ content are higher than previous reported CDs. ${ }^{39}$ It is suggested that there is more nanosheet in carbon core, resulting in more $\mathrm{N}$ atom could enter carbon core. Herein, quantum yields of as-prepared CDs are higher than previous CDs. The band at $1396.46 \mathrm{~cm}^{-1}$ corresponding to C-N became more obvious in the FT-IR spectra. It is indicated that $\mathrm{N}$ atom gradually substituted $\mathrm{O}$ atom during the carbonization process, leading to $\mathrm{N}$ content increase as XPS data. From the composition and structural of CDs studies, we can see that the $\mathrm{N}$ content and the $\mathrm{C}-\mathrm{N}$ formation were critical to improve the quantum yields of CDs.

\section{Spectral characteristics of CDs}

The UV-Vis absorption spectrum of the CDs is shown in Fig. 4a. The CDs exhibited a strong absorption in the UV region, and the

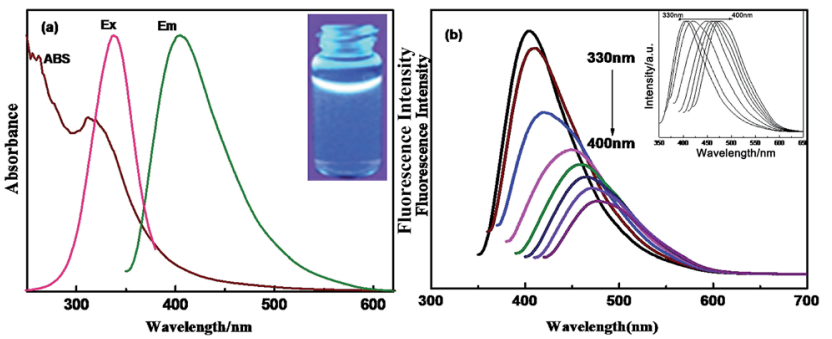

Fig. 4 Spectral characteristics of the as-prepared CDs. (a) UV-vis absorption spectrum and fluorescence spectrum of the CDs. (b) Emission spectrum of the carbon spot with the excitation wavelength increased from $330 \mathrm{~nm}$ to $400 \mathrm{~nm}$ at $10 \mathrm{~nm}$ intervals. The inset figure shows the normalized emission spectra.

absorption range extends to the tail of the visible region. A small shoulder peak caused by a $\pi-\pi^{*}$ transition was observed at $267 \mathrm{~nm}$, indicating that the CDs contained functional groups such as carboxyl groups and other isolated pairs of electrons. There is a very broad absorption peak at $314 \mathrm{~nm}$. The CDs showed a relatively symmetrical excitation and emission spectrum, the maximum excitation wavelength was $360 \mathrm{~nm}$, and the maximum emission wavelength was $425 \mathrm{~nm}$. Under a $365 \mathrm{~nm}$ UV lamp, the aqueous solution of CDs showed a blue fluorescence (inset figure in Fig. 4a).

Meanwhile, the emission peak of the CDs changed with the excitation wavelength. When the excitation wavelength increased from $360 \mathrm{~nm}$ to $500 \mathrm{~nm}$ at $10 \mathrm{~nm}$ intervals, the emission peak red shifted accompanied by a decrease in the fluorescence intensity. This might be because of the different emission sites on the surface or because of the nonuniformity of the particle size (quantum size effect). The excellent luminescent properties of CDs should be closely related to the quantum yield. The quantum yield of the CDs was as high as $61.1 \%$ (Fig. S2 $\dagger$ ) at an excitation wavelength of $360 \mathrm{~nm}$ using quinine as a reference. Such a high quantum yield can be attributed to the successful nitrogen doping, showing that nitrogen doping is an effective way to improve the quantum yield of CDs.

\section{Stability of the CDs}

As shown in Fig. S3a, $\uparrow$ the experimental results prove that the CDs have high photostability against photobleaching. When the aqueous solution of CDs was continuously irradiated with a $360 \mathrm{~nm}$ excitation laser for $30 \mathrm{~min}$, there was almost no change in the fluorescence intensity, and no light blinking phenomenon occurred. Meanwhile, the CDs also exhibited good thermal stability and maintained their stability at different temperatures. Additionally, with an increase in temperature, the fluorescence intensity of the CDs was barely affected (Fig. S3b $\dagger$ ). As shown in Fig. S3c, $\dagger$ the CDs were also stable in DMF, DMSO, ethanol, and other common organic solvents, and this is very important for the application of CDs in actual complex systems. Moreover, the CDs also showed a strong resistance to salt (Fig. S3d $\dagger$ ). The fluorescence intensity of the CDs barely changed within the salt concentrations of 0 to $4 \mathrm{M}$, and even higher ionic strength did not cause aggregation. 
The good stabilities of the CDs shown above provide a good foundation for practical applications.

\section{Binding of the as-prepared CDs with metal ions}

The as-prepared CDs have rich multifunctional groups, such as carboxyl and hydroxyl, which can easily bind with metal ions. In order to investigate the binding features of CDs, we examined the effects of different metal ions on the fluorescence of the CDs under the same conditions and studied the selectivity of the CDs for the metal ions. Fig. 5a shows the effects of 16 different metal ions, including $\mathrm{Ag}^{+}, \mathrm{Al}^{3+}, \mathrm{Ca}^{2+}, \mathrm{Cd}^{2+}, \mathrm{Co}^{2+}, \mathrm{Cu}^{2+}, \mathrm{Cr}^{3+}$, $\mathrm{Hg}^{2+}, \mathrm{K}^{+}, \mathrm{Mg}^{2+}, \mathrm{Pb}^{2+}$, and $\mathrm{Fe}^{3+}$, on the fluorescence intensity of the reaction system. The concentrations of the metal ions were $125 \mu \mathrm{M} . F_{0}$ represents the fluorescence intensity of the solution of CDs without the addition of metal ions, and $F$ represents the fluorescence intensity of the mixed solution after the addition of the metal ions. The experimental results indicate that the fluorescence of CDs could be specifically quenched by $\mathrm{Fe}^{3+}$. In contrast, other metal ions had little influence on the fluorescence intensity of the CDs. Although the fluorescence intensities of the CDs were slightly reduced in the presence of some metal ions, the degrees of reduction were all very weak.

This binding mechanism, which the as-prepared CDs have specific binding ability with $\mathrm{Fe}^{3+}$, was most likely because of the presence of hydroxyl and carboxyl functional groups on the surface of the CDs. It is known that $\mathrm{Fe}^{3+}$ is able to coordinate with hydroxyl groups on edges of CDs and the binding constant of $\mathrm{Fe}^{3+}$ with hydroxyl is higher than other metal ions, ${ }^{52-54}$ so we supposed that $\mathrm{Fe}^{3+}$ binding with hydroxyl groups on the surface of CDs to form metal hydroxides or other complexes, resulting in the aggregation of CDs. It is well known, $\mathrm{Fe}^{3+}$ is easily hydrolyzed even in acidic solution. ${ }^{55,56}$ The aggregation of CDs, which resulted in fluorescence quenching, might be caused by the hydrolysis of $\mathrm{Fe}^{3+}$. In order to investigate the hydrolysis effect for the detection of $\mathrm{Fe}^{3+}$, the $\mathrm{pH}$ response of CDs was explored. As Fig. S4† shown, the CDs had high stability against $\mathrm{pH}$ variation in the range of 3-12. Moreover, as illustrated in Fig. S5 and $\mathrm{S} 6, \uparrow \mathrm{Fe}^{3+}$ hydrolysis has no obvious effect on sensing experiments in HAc-NaAc buffer with $\mathrm{pH}$ 3.2. To further investigate the sensing mechanism, we tested fluorescence lifetime of CDs before and after addition of $\mathrm{Fe}^{3+}$. As Fig. S7†
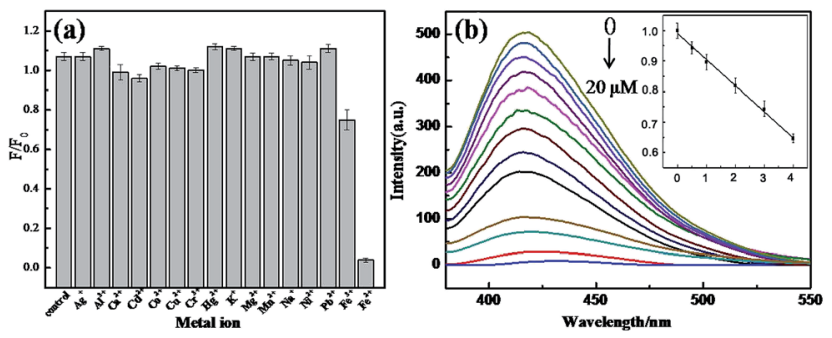

Fig. 5 Investigation of the sensitivity and selectivity in $\mathrm{Fe}^{3+}$ detection. (a) The influence of metal ions on the fluorescence intensity of the CDs. The concentration of metal ions was $125 \mu \mathrm{M} \mathrm{L}^{-1}$. (b) The fluorescence intensity of the $\mathrm{CDs}$ varied with the $\mathrm{Fe}^{3+}$ concentration, and the concentration of $\mathrm{Fe}^{3+}$ was in the range from 0 to $20 \mu \mathrm{M}$. The inset figure shows the linear relationship from 0.5 to $4 \mu \mathrm{M}$. shown, fluorescence lifetime of CDs had no obvious decreased upon addition of $\mathrm{Fe}^{3+}$. It was demonstrated that fluorescence quenching of CDs caused by the aggregation of CDs upon addition of $\mathrm{Fe}^{3+}$, instead of electron transfer or fluorescence resonance energy transfer. As Fig. $\mathrm{S} 8 \uparrow$ shown, the average size of the as-prepared CDs increased from $5.6 \mathrm{~nm}$ to $13.8 \mathrm{~nm}$ when $\mathrm{Fe}^{3+}$ ions have interacted with CDs. It was indicated that the aggregation of CDs have taken placed, resulting in fluorescence quenching of the CDs.

\section{Detection of the concentration of $\mathrm{Fe}^{3+}$}

It is noticeable that $\mathrm{Fe}^{3+}$ can induce the fluorescence quenching of CDs, which can be used to detect $\mathrm{Fe}^{3+}$. As Fig. $5 \mathrm{~b}$ shown, the fluorescence intensity of the CDs decreased with increasing the concentration of $\mathrm{Fe}^{3+}$ when $\mathrm{Fe}^{3+}$ was added to the system. This was because of the interactions between $\mathrm{Fe}^{3+}$ and the hydroxyl groups on the surface of CDs as they were added to the system, which resulted in the aggregation of CDs and thus in the fluorescence quenching. In addition, for concentrations of $\mathrm{Fe}^{3+}$ in the range of $0.5-4 \mu \mathrm{M}$, when the $\mathrm{Fe}^{3+}$ concentration increased, the degree of fluorescence quenching increased, and there was a good linear relationship (inset figure in Fig. $5 \mathrm{~b}$ ). $F_{0}$ represents the fluorescence intensity of the CDs without the addition of $\mathrm{Fe}^{3+}$, and $F$ represents the fluorescence intensity of the mixture after the addition of $\mathrm{Fe}^{3+}$. The linear regression equation was $F /$ $F_{0}=0.998-0.037 c$ (regression coefficient $R^{2}=0.998$ ), and the detection limit $(3 \sigma)$ was calculated to be $0.38 \mu \mathrm{M}$.

\section{Conclusions}

In this work, Bombyx mori silk was selected as the raw material, and nitrogen-doped blue fluorescent CDs that had high quantum yield were synthesized using a one-step hydrothermal method. The method was mild, environmental friendly, and economical. The obtained CDs showed the characteristics of nitrogen doping and exhibited excellent optical properties and good stability. In addition, the CDs can maintain photostability in the presence of strong acid or base, high salt concentrations, and most organic solvents. Based on the interaction between $\mathrm{Fe}^{3+}$ and the hydroxyl groups on the surface of $\mathrm{CDs}, \mathrm{Fe}^{3+}$ ions can induce fluorescence quenching of the CDs, thus making the CDs a luminescent probe for selective and sensitive detection of $\mathrm{Fe}^{3+}$ ions.

\section{Conflicts of interest}

There are no conflicts to declare.

\section{Acknowledgements}

This work was financially supported by the National Natural Science Foundation of China (NSFC, No. 21535006, No. 21705132), Chongqing Research Program of Basic Research and Frontier Technology (No. cstc2017jcyjAX0060), as well as the Fundamental Research Funds for the Central Universities (XDJK2017C065, XDJK2017B056). 


\section{Notes and references}

1 X. Xu, R. Ray, Y. Gu, H. J. Ploehn, L. Gearheart, K. Raker and W. A. Scrivens, J. Am. Chem. Soc., 2004, 126, 12736-12737.

2 S. Y. Lim, W. Shen and Z. Gao, Chem. Soc. Rev., 2015, 44, 362381.

3 J. Zhou, Y. Yang and C. Y. Zhang, Chem. Rev., 2015, 115, 11669-11717.

4 Q. Xu, Y. Zhang, B. Tang and C. Y. Zhang, Anal. Chem., 2016, 88, 2051-2058.

5 U. Resch-Genger, M. Grabolle, S. Cavaliere-Jaricot, R. Nitschke and T. Nann, Nat. Methods, 2008, 5, 763-775.

6 S.-T. Yang, L. Cao, P. G. Luo, F. Lu, X. Wang, H. Wang, M. J. Meziani, Y. Liu, G. Qi and Y.-P. Sun, J. Am. Chem. Soc., 2009, 131, 11308-11309.

7 S. Zhu, Q. Meng, L. Wang, J. Zhang, Y. Song, H. Jin, K. Zhang, H. Sun, H. Wang and B. Yang, Angew. Chem., Int. Ed., 2013, 125, 4045-4049.

8 G. Hong, S. Diao, A. L. Antaris and H. Dai, Chem. Rev., 2015, 115, 10816-10906.

9 C.-W. Lai, Y.-H. Hsiao, Y.-K. Peng and P.-T. Chou, J. Mater. Chem., 2012, 22, 14403-14409.

10 S. Hamd-Ghadareh, A. Salimi, F. Fathi and S. Bahrami, Biosens. Bioelectron., 2017, 96, 308-316.

11 C. Lan, L. Zhang, B. Shi, D. Chen, L. Zhao and S. Zhao, J. Mater. Chem. B, 2017, 5, 5265-5271.

12 A. Emam, S. A. Loutfy, A. A. Mostafa, H. Awad and M. B. Mohamed, RSC Adv., 2017, 7, 23502-23514.

13 X. Huang, F. Zhang, L. Zhu, K. Y. Choi, N. Guo, J. Guo, K. Tackett, P. Anilkumar, G. Liu and Q. Quan, ACS Nano, 2013, 7, 5684-5693.

14 T. Feng, X. Ai, G. An, P. Yang and Y. Zhao, ACS Nano, 2016, 10, 4410-4420.

15 M. Zhang, N. Zhou, P. Yuan, Y. Su, M. Shao and C. Chi, RSC Adv., 2017, 7, 9284-9293.

16 Z. X. Liu, B. B. Chen, M. L. Liu, H. Y. Zou and C. Z. Huang, Green Chem., 2017, 19, 1494-1498.

17 S. Bhattacharyya, B. Konkena, K. Jayaramulu, W. Schuhmann and T. K. Maji, J. Mater. Chem. A, 2017, 5, 13573-13580.

18 Q. Li, M. Zhou, Q. Yang, Q. Wu, J. Shi, A. Gong and M. Yang, Chem. Mater., 2016, 28, 8221-8227.

19 B. B. Chen, Z. X. Liu, W. C. Deng, L. Zhan, M. L. Liu and C. Z. Huang, Green Chem., 2016, 18, 5127-5132.

20 M. M. Al Awak, P. Wang, S. Wang, Y. Tang, Y.-P. Sun and L. Yang, RSC Adv., 2017, 7, 30177-30184.

21 M. Lin, H. Y. Zou, T. Yang, Z. X. Liu, H. Liu and C. Z. Huang, Nanoscale, 2016, 8, 2999-3007.

22 T. Skaltsas, M. Goulielmaki, A. Pintzas, S. Pispas and N. Tagmatarchis, J. Mater. Chem. B, 2017, 5, 5397-5402.

23 M. X. Gao, L. Yang, Y. Zheng, X. X. Yang, H. Y. Zou, J. Han, Z. X. Liu, Y. F. Li and C. Z. Huang, Chem.-Eur. J., 2017, 23, 2171-2178.

24 Y. H. Yuan, Z. X. Liu, R. S. Li, H. Y. Zou, M. Lin, H. Liu and C. Z. Huang, Nanoscale, 2016, 8, 6770-6776.
25 M. Havrdova, K. Hola, J. Skopalik, K. Tomankova, M. Petr, K. Cepe, K. Polakova, J. Tucek, A. B. Bourlinos and R. Zboril, Carbon, 2016, 99, 238-248.

26 Z. Wang, P. Long, Y. Feng, C. Qin and W. Feng, RSC Adv., 2017, 7, 2810-2816.

27 R. S. Li, P. F. Gao, H. Z. Zhang, L. L. Zheng, C. M. Li, J. Wang, Y. F. Li, F. Liu, N. Li and C. Z. Huang, Chem. Sci., 2017, 68296835.

28 X. Gao, C. Ding, A. Zhu and Y. Tian, Anal. Chem., 2014, 86, 7071-7078.

29 J. Zhou, C. Booker, R. Li, X. Zhou, T.-K. Sham, X. Sun and Z. Ding, J. Am. Chem. Soc., 2007, 129, 744-745.

30 H. Liu, T. Ye and C. Mao, Angew. Chem., Int. Ed., 2007, 46, 6473-6475.

31 Y.-P. Sun, B. Zhou, Y. Lin, W. Wang, K. S. Fernando, P. Pathak, M. J. Meziani, B. A. Harruff, X. Wang and H. Wang, J. Am. Chem. Soc., 2006, 128, 7756-7757.

32 Y. Dong, R. Wang, H. Li, J. Shao, Y. Chi, X. Lin and G. Chen, Carbon, 2012, 50, 2810-2815.

33 J. Du, Y. Zhao, J. Chen, P. Zhang, L. Gao, M. Wang, C. Cao, W. Wen and C. Zhu, RSC Adv., 2017, 7, 33929-33936.

34 S. Zhu, Q. Meng, L. Wang, J. Zhang, Y. Song, H. Jin, K. Zhang, H. Sun, H. Wang and B. Yang, Angew. Chem., Int. Ed., 2013, 52, 1-6.

35 H. Tetsuka, R. Asahi, A. Nagoya, K. Okamoto, I. Tajima, R. Ohta and A. Okamoto, Adv. Mater., 2012, 24, 5333-5338.

36 B. Martindale, G. A. Hutton, C. A. Caputo, S. Prantl, R. Godin, J. R. Durrant and E. Reisner, Angew. Chem., Int. Ed., 2017, 56, 6459-6463.

37 Q. Xiao, Y. Liang, F. Zhu, S. Lu and S. Huang, Microchim. Acta, 2017, 184, 2429-2438.

38 N. C. Tansil, Y. Li, C. P. Teng, S. Zhang, K. Y. Win, X. Chen, X. Y. Liu and M.-Y. Han, Adv. Mater., 2011, 23, 1463-1466.

39 Z. L. Wu, P. Zhang, M. X. Gao, C. F. Liu, W. Wang, F. Leng and C. Z. Huang, J. Mater. Chem. B, 2013, 1, 2868-2873.

40 Y. Choi, B. Kang, J. Lee, S. Kim, G. T. Kim, H. Kang, B. R. Lee, H. Kim, S.-H. Shim, G. Lee, O.-H. Kwon and B.-S. Kim, Chem. Mater., 2016, 28, 6840-6847.

41 D. Pan, J. Zhang, Z. Li, C. Wu, X. Yan and M. Wu, Chem. Commun., 2010, 46, 3681-3683.

42 Y. Dong, J. Shao, C. Chen, H. Li, R. Wang, Y. Chi, X. Lin and G. Chen, Carbon, 2012, 50, 4738-4743.

43 L. Yang, W. Jiang, L. Qiu, X. Jiang, D. Zuo, D. Wang and L. Yang, Nanoscale, 2015, 7, 6104-6113.

44 Y.-P. Sun, B. Zhou, Y. Lin, W. Wang, K. A. S. Fernando, P. Pathak, M. J. Meziani, B. A. Harruff, X. Wang, H. Wang, P. G. Luo, H. Yang, M. E. Kose, B. Chen, L. M. Veca and S.-Y. Xie, J. Am. Chem. Soc., 2006, 128, 7756-7757.

45 F. Zhang, X. Feng, Y. Zhang, L. Yan, Y. Yang and X. Liu, Nanoscale, 2016, 8, 8618-8632.

46 S. Qu, X. Wang, Q. Lu, X. Liu and L. Wang, Angew. Chem., Int. Ed., 2012, 124, 12381-12384.

47 D. Qu, M. Zheng, P. Du, Y. Zhou, L. Zhang, D. Li, H. Tan, Z. Zhao, Z. Xie and Z. Sun, Nanoscale, 2013, 5, 12272-12277. 48 Y. Song, S. Zhu, S. Zhang, Y. Fu, L. Wang, X. Zhao and B. Yang, J. Mater. Chem. C, 2015, 3, 5976-5984. 
49 D. Qu, M. Zheng, L. Zhang, H. Zhao, Z. Xie, X. Jing, R. E. Haddad, H. Fan and Z. Sun, Sci. Rep., 2014, 4, 5294.

50 Y. Zhang, X. Liu, Y. Fan, X. Guo, L. Zhou, Y. Lv and J. Lin, Nanoscale, 2016, 8, 15281-15287.

51 C. Sun, Y. Zhang, P. Wang, Y. Yang, Y. Wang, J. Xu, Y. Wang and W. W. Yu, Nanoscale Res. Lett., 2016, 11, 110.

52 Q. Xu, P. Pu, J. Zhao, C. Dong, C. Gao, Y. Chen, J. Chen, Y. Liu and H. Zhou, J. Mater. Chem. A, 2015, 3, 542-546.
53 K. Qu, J. Wang, J. Ren and X. Qu, Chem.-Eur. J., 2013, 19, 7243-7249.

54 J. Ju and W. Chen, Biosens. Bioelectron., 2014, 58, 219-225.

55 S. Musić, S. Krehula, S. Popović and Ž. Skoko, Mater. Lett., 2003, 57, 1096-1102.

56 X. Hu, J. C. Yu, J. Gong, Q. Li and G. Li, Adv. Mater., 2007, 19, 2324-2329. 